\title{
First steps to obtaining primary cell cultures of different tissues of endemic Baikal amphipods Eulimnogammarus verrucosus
}

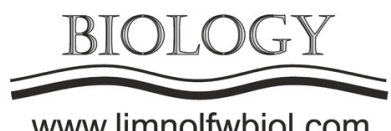

www.limnolfwbiol.com

\author{
Nazarova A.A., Gurkov A.N., Vereshchagina K.P., Mutin A.D., Timofeyev M.A. \\ Irkutsk State University, 664025, Lenin str. 3, Irkutsk, Russia
}

\begin{abstract}
In this study we tested two methods of cell isolation for obtaining primary cultures of muscle and ovarian cells from endemic Baikal amphipods Eulimnogammarus verrucosus. The method of tissue explants was found superior to the enzymatic disaggregation in terms of cell survival for both tissues, but muscle cells generally had low viability. Free ovarian cells retained their viability at the level of $70 \%$ for at least 4 hours in the L-15 medium with 10\% FBS, which demonstrates their possible suitability for further cultivation.
\end{abstract}

Keywords: Baikal, amphipods, primary cell cultures, enzymatic disaggregation, explants.

\section{Introduction}

Lake Baikal is the largest freshwater lake with a high level of endemism in invertebrates. Endemic Baikal amphipods (Amphipoda, Crustacea) are one of the key components of the lake ecosystem (Takhteev, 2000), and they demonstrate substantial differences in adaptive potential to various stressors in comparison to their Holarctic counterparts (Protopopova et al., 2014; Axenov-Gribanov et al., 2016). Primary cell cultures are an important tool for detailed study of the molecular foundations of the functioning of any multicellular organism and can be used as a model for various purposes in ecophysiological and ecotoxicological research. However, despite the importance of amphipods for the food webs of Baikal and their unique features, the techniques for the preparation of primary cell cultures of these endemics remain poorly developed. The aim of this study was to develop methods for obtaining primary ovary and muscle cell cultures of endemic Eulimnogammarus verrucosus (Gerstfeldt, 1858), one of the most widespread amphipod species in Baikal littoral.

\section{Materials and methods}

Before tissue excision, amphipods were euthanized in $0.05 \%$ clove oil solution for 10 minutes. Animals were surface-sterilized by submersion in sterile physiological solution for 5-10 minutes and then washed in $70 \%$ ethanol (Han et al., 2013). The ovaries and muscles were excised with sterile scissors and tweezers, washed in the buffer and cut into small pieces of approximately $0.1 \mathrm{~mm}$. For enzymatic tissues disaggregation, we used exposure in $0.25 \%$ trypsin solution for either 5 or 15 minutes. The obtained homogenate was washed from trypsin by centrifugation for 5 minutes at $2000 \mathrm{~g}$ several times. For testing the explant method, pieces of muscles and ovaries were placed in a number of cultural media: Grace's medium, Leibovitz L-15 medium and modified Leibovitz L-15 medium (ML-15, $2 \times$ concentrated) supplemented with no, $5 \%, 10 \%$ or $15 \%$ fetal bovine serum (FBS). Quantitative assessment of cell migration from explants in different culture media was carried out the day after. Cell viability was evaluated using $0.4 \%$ trypan blue staining.

\section{Results and discussion}

Animal cells can be extracted from tissues using either enzymatic disaggregation or spontaneous migration from tissue explants, and both methods were tested in the current study. Trypsin disaggregation of the muscles dissected from the amphipod pereon was effective (Fig. 1A), but further evaluation of the cell viability was not successful. In particular, we could not locate the nuclei of the cells that are usually stained with trypan blue after cell death, and, even more importantly, all cells were stained. The explant method demonstrated slightly higher overall effectiveness. After a day of incubation in the used culture media of the muscle explants from the basal part of the limbs (Fig. 1B), migration of branched structures was observed (Fig. 1C). However, staining of these structures with trypan blue showed some but still low viability. Thus, 


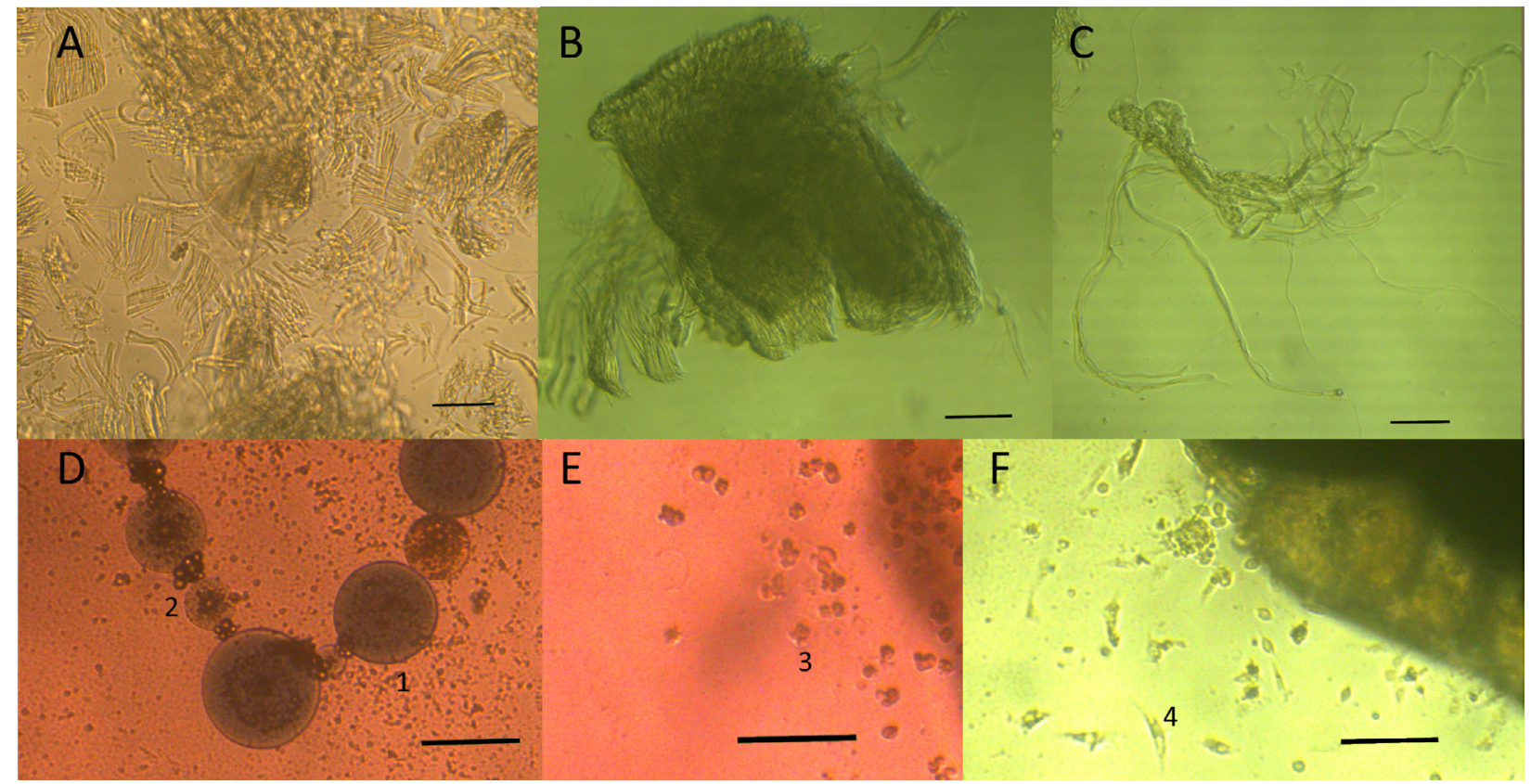

Fig.1. A - Amphipod muscle tissue after treatment with $0.25 \%$ trypsin solution for 15 minutes. B - Amphipod muscle explants. C - Migrated structure. D-F - The ovarian cells. The line is 100 microns.

amphipod muscle cells seem to be unsuitable for cultivation.

The ovarian cells demonstrated much higher potential for obtaining the primary cultures. We observed migration of four cell types (Fig. 1D-1F): large round cells, probably an egg cell; clusters of 6-8 small round cells without obvious morphology; irregularly shaped small cells attached to the plate bottom; small fibroblast-like cells with apparent nucleus, potentially hemocytes. The largest number of cells migrated in L-15 and ML-15 with any amount of FBS. Four hours after media exchange the ovarian cells demonstrated the highest survival in the L-15 medium with 10\% FBS (70\%). Trypsin treatment during $5 \mathrm{~min}$ allowed us to extract only cells of the second type, but also with relatively good viability up to $60 \%$.

\section{Findings}

According to the results of using both the explant method and the method of enzymatic disaggregation, it should be concluded that amphipod muscle cells have low potential for obtaining primary cultures. On the contrary, ovarian cells are more appropriate for this purpose, especially with application of the explant method and the L15 medium.

\section{Acknowledgments}

This work was supported be the grants from the Russian Foundation for Basic Research (№19-54-04008) and the Russian Science Foundation (№18-44-06201).

\section{References}

Axenov-Gribanov D., Bedulina D., Shatilina Zh. et al. 2016. Thermal preference ranges correlate with stable signals of universal stress markers in Lake Baikal endemic and holarctic amphipods. PlosOne 11. DOI: 10.1371/journal. pone. 0164226

Han Q., Li P., Lu X. et al. 2013. Improved primary cell culture and subculture of lymphoid organs of the greasyback shrimp Metapenaeus ensis. Aquaculture 410-411: 101-113. DOI: 10.1016/j.aquaculture.2013.06.024

Protopopova M.V., Pavlichenco V.V., Menzel R. et al. 2014. Contrasting cellular stress responses of Baikalian and Palearctic amphipods upon exposure to humic substances: environmental implications. Environmental Science and Pollution Research 21: 14124-1437. DOI: 10.1007/ s11356-014-3323-8

Takhteev V.V. 2000. Trends in the evolution of Baikal amphipods and evolutionary parallels with some marine malacostracan faunas. Advances in Ecological Research 31: 196-220. DOI: 10.1016/S0065-2504(00)31013-3 